Ahmed K. Al-Jaberi, Aras Asaad, Sabah A. Jassim, and Naseer Al-Jawad "Topological data analysis to improve exemplar-based inpainting", Proc. SPIE 10668, Mobile Multimedia/Image Processing, Security, and Applications 2018, 1066805 (14 May 2018); https://doi.org/10.1117/12.2309931

Copyright 2018 Society of Photo-Optical Instrumentation Engineers (SPIE). One print or electronic copy may be made for personal use only. Systematic reproduction and distribution, duplication of any material in this publication for a fee or for commercial purposes, and modification of the contents of the publication are prohibited. 
Aras T. Asaad, Rasber Dh. Rashid, and Sabah A. Jassim "Topological image texture analysis for quality assessment", Proc. SPIE 10221, Mobile Multimedia/Image

Processing, Security, and Applications 2017, 102210l (10 May

2017); https://doi.org/10.1117/12.2268471

Copyright 2017 Society of Photo-Optical Instrumentation Engineers (SPIE). One print or electronic copy may be made for personal use only. Systematic reproduction and distribution, duplication of any material in this publication for a fee or for commercial purposes, and modification of the contents of the publication are prohibited. 


\title{
Topological Image Texture Analysis for Quality Assessment
}

\author{
Aras T. Asaad ${ }^{1}$, Rasber Dh. Rashid ${ }^{2}$ and Sabah A. Jassim ${ }^{1}$ \\ ${ }^{1}$ Applied Computing Department, The University of Buckingham, UK. \\ ${ }^{2}$ Software Engineering Department, Koya University, Iraq. \\ \{aras.asaad, sabah.jassim\}@buckingham.ac.uk, rasber.rashid@koyauniversity.org
}

\begin{abstract}
Image quality is a major factor influencing pattern recognition accuracy and help detect image tampering for forensics. We are concerned with investigating topological image texture analysis techniques to assess different type of degradation. We use Local Binary Pattern (LBP) as a texture feature descriptor. For any image construct simplicial complexes for selected groups of uniform LBP bins and calculate persistent homology invariants (e.g. number of connected components). We investigated image quality discriminating characteristics of these simplicial complexes by computing these models for a large dataset of face images that are affected by the presence of shadows as a result of variation in illumination conditions. Our tests demonstrate that for specific uniform LBP patterns, the number of connected component not only distinguish between different levels of shadow effects but also help detect the infected regions as well.
\end{abstract}

Keywords: Image Quality, Local Binary Pattern, Simplicial complexes.

\section{INTRODUCTION}

Digital images are subject to a many distortions while processing, transmission, storing and compressing which will result in many degradation types in visual quality. Digital image processing, in general, can change the appearance of images in a way that human visual system will judge the change either beneficial or injuriously. Therefore, image quality assessment plays an important role in many digital image processing applications such as biomedical imaging, segmentation, acquisition image fusion and printing and displaying systems. Also, image quality is a crucial factor affecting the accuracy of pattern recognition and help detect image manipulation for forensics. In general, there are two types of image quality assessment; subjective and objective [1]. In many multimedia applications, subjective evaluation is the most reliable and accurate method to assess the image quality because human observers are the final users. However, subjective assessment is time-consuming and expensive which makes them to be inconvenient in real-world applications. Objective image quality assessment, on the other hand, tries to dynamically evaluate and adjust image quality. Peak Signal-to-Noise-Ration (PSNR) and Mean squared Error (MSE) are the most widely used image distortion/ quality assessment measures [2]. Despite the interesting points about these two measures, they have been widely criticized for not being able to correlate well with perceived quality measurement as well as not being able to locate degraded image regions/locations. In the last four decades, a wide range of researches have been conducted to develop a new image quality measurement that take advantage of human visual system characteristics. Objective image quality measurement can be classified into three categories based 
on the availability of original distortion-free image. Full-reference, where a complete reference image is available for assessments, no-reference or 'blind' category we don't have access to the original/reference image (i.e. original/reference image is not available), and the third category is known as reduced-reference where a reference image is only available partially as some features of the reference image will be extracted and employed as information to measure the quality of the test image [2] [1]. No-reference image quality assessment is convenient in practice because in many real-world applications the reference image is not available.

This paper intends to describe a new approach to image quality assessment, based on topological image texture analysis. In particular, we use local binary pattern (LBP) as a texture feature descriptor and then constructing simplicial complexes for selected group of uniform LBP bins as well as calculating persistent homology invariant ( e.g number of connected components). We tested this approach on large data set of face images that are affected by presence of shadow as a result of variation in illumination conditions. For specific uniform LBP patterns, the number of connected components successfully separated different levels of shadow effects as well as detected the infected regions through simplicial complexes.

The rest of the paper is organised as follows. Section 2 presents the fundamentals of LBP and how the LBP is used. Section 3 presents the idea of simplicial complexes and the way of how it conducted on the LBP images. Section 4 presents the proposed image quality assessment scheme, while section 5 presents the experimental data and the results of our evaluation. Finally our conclusions are presented in section 6.

\section{LOCAL BINARY PATTERNS (LBP)}

Local Binary pattern is a visual descriptor which has been first introduced by Ojala et al. [3]. The core idea of LBP is: substituting each pixel in the given image by an eight bit binary code which will be deduced from the neighbouring pixels. For the sake of clarity, consider a $3 \times 3$ block matrix taken from an image as shown in figure (1).

Then the value of the pixel in the center of the matrix will be subtracted from the 8 neighbouring pixels, and 0 or 1 will be assigned to a bit depending on the result of the subtraction procedure. Resulting bits from the subtraction operation for 8 neighbouring pixels will be concatenated and encoded into binary strings in clockwise direction. Obtained binary strings will be called Local Binary Patterns or sometimes LBP codes. The procedure for converting the decimal value of the central pixel $\left(x_{c}, y_{c}\right)$, i.e. 32 , into LBP form will be as follow:

$$
\operatorname{LBP}\left(x_{c}, y_{c}\right)=\sum_{i=0}^{i=7} \alpha\left(P_{i}-P_{c}\right) 2^{i}
$$

where $P_{i}$ is the neighbouring grey value pixels, $P_{c}$ is the centre grey value pixel and the function $\alpha(x)$ is as follow:

$$
\alpha(x)= \begin{cases}1 & \text { if } x \geq 0 \\ 0 & \text { if } x<0\end{cases}
$$

Applying above procedure on the block matrix will result in getting the binary string $00001111($ decimal = 15) as follow: 


\begin{tabular}{|c|c|c|}
\hline 72 & 67 & 32 \\
\hline 11 & 32 & 44 \\
\hline 30 & 17 & 20 \\
\hline
\end{tabular}
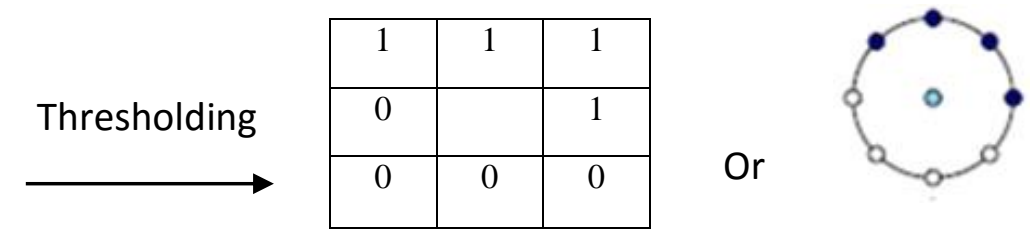

Figure (1): An example of Local Binary Operator

When the image of interest is converted into a local binary pattern form, then a histogram of the image will be calculated. Local detailed information, such as edges, lines, spots and flat regions can be detected by the LBP histogram [4] . It has been shown that certain patterns of LBP operator contain more information than others. Ojala et al. named these patterns as uniform patterns. The texture of an image can be described through LBP operator by only using $2^{p}$ binary patterns. A circular bit string is considered to be uniform if it contains at most two bitwise transitions from 1 to 0 and from 0 to 1 . For the sake of clarity, an example of uniform bins will be 11111111 (zero transition) and 00111111 (two transitions) whereas non-uniform patterns will be like 10101010 (eight transitions). Ojala et al. experimentally demonstrated that $90.6 \%$ of all patterns using $(8,1)$ neighbouring pixels in texture images are uniform patterns. In facial images, [5] are the first who applied the idea of uniform LBP.

It is easy to show that within the $(8,1)$ neighbourhood, there are only 58 uniform LBP-patterns, and the traditional uniform LBP histogram consists of 59 bins accounting for the 58 uniform patterns and one bin that corresponds to the summation of non-uniform patterns [6]. Researchers are taking histograms of LBP-image as a feature for the classification and recognition purposes [7] [8].

In this paper, we focus on the geometry of the 58 uniform bins such that after we apply LBP operator on the image, we construct the simplicial complex for each of the 58 uniform bins. Furthermore, we grouped the LBP-Uniform bins into seven groups according to their binary code pattern excluding 0000000 and 11111111 . The seven groups are shown in figure (2), where we ordered the rotations of the binary code patterns. In the next section we will demonstrate that separating the bins according to their binary code patterns will reveal the presence of some previously unseen textures by comparing the number of connected components. The simplicial complexes constructed from the bins shows some image textures which convey useful information about the images and at the same time it is hard to detect those features by other methods.

\section{TOPOLOGICAL DATA/IMAGE ANALYSIS}

One of the primary goals of topology is to classify objects (shapes) according to their connectivity using a finite combinatorial process known as simplicial complexes. The fundamental idea behind using topology for data analysis is that via topology, one can extract shapes, or patterns, from complex high dimensional data sets and then obtaining deep intuitive understanding about them. Extracting patterns (or shapes) via topology is possible because topology study shapes in a coordinate free, invariant under small deformation and in a compressed representation [9]. These three important properties of topology have been discussed in detail in [9]. The application scope of topological data analysis includes, but 
is not limited to, gait recognition [10] [11] [12], brain artery [13], hurricane and galaxies analysis [14], dimensionality reduction schemes evaluation [15], classification of hepatic lesions [16], shape classification using LBP and persistent [17].

Persistent homology is an invariant that characterizes the topological properties of objects (topological spaces). Homology measures the number of connected components, loops, voids and so on of shapes and then can be used to differentiate distinct spaces and objects from one another. The number of connected components generate zero homology groups associated with simplicial complexes. This paper will focus on computing persistent homology of simplicial complexes which will be constructed from a selected group of uniform local binary patterns. To be specific, the type of simplicial complex which will be built is known as Vietoris-Rips complex (or Rips complex).

In order to construct Rips complex for point cloud data (specific uniform LBP bins), one needs to select a threshold parameter $T$ as a first step. Then by gradually increasing $T$, high dimensional simplices will be constructed. For sufficiently small $T$, only zero dimensional simplices will be obtained and for sufficiently large $T$, a single high dimensional simplex will be constructed. The features that are remain after changing the threshold are considered to be the features that are conveying true information about the data whereas the features that are not persistent by increasing the threshold gradually considered to be noise [18] [19]. This approach is known as persistent homology where at each threshold homology invariants will be computed for different quality of face images.

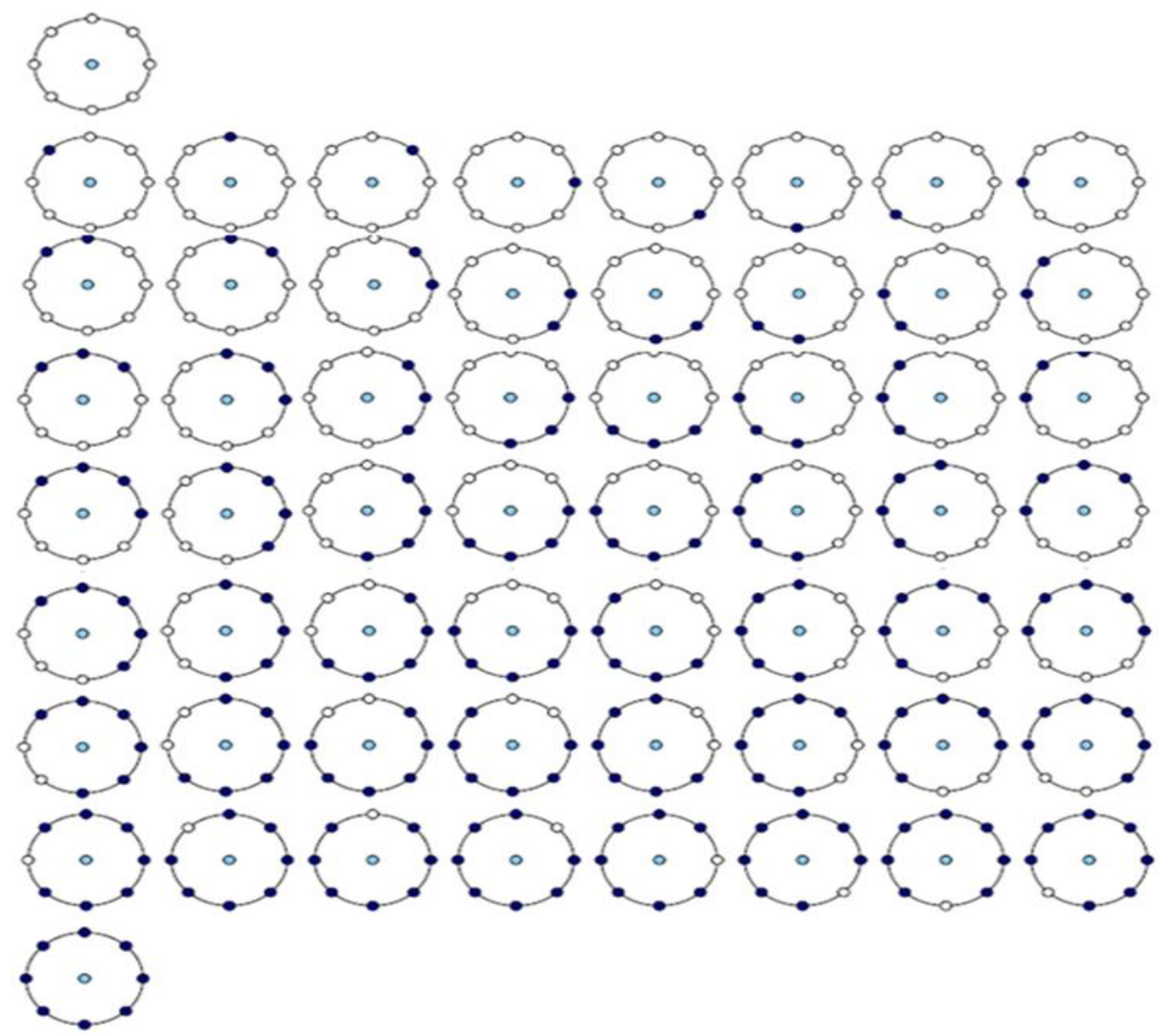

Figure (2): The 58 different uniform patterns in $(8, \mathrm{R})$ neighbourhood. 


\section{PROPOSED SCHEME}

We assessing the quality of images, face images in our case, based on computing the number of connected components of face images after transforming them into LBP space. First, we transform the image into a local binary pattern space because we want to partition the image into small parts (i.e. bins) which enables simplicial complex (SC) construction easy in comparison with trying to build simplicial complex from the whole image. After this transformation, we only select the LBP-Uniform bins. In order to be able to construct the simplicial complex for each bin, we need to determine a way of selecting a suitable threshold $T=\left[T_{\min }, T_{\max }\right]$ to capture the topology in each bin. To do so, we compute the distance between points in each of the 56 uniform bins (excluding 00000000 and 11111111 pattern code). We then sort the minimum and the maximum distances between the points in two arrays. From the minimum array, which contain 56 minimum distances, we select the maximum of the minimum distances to be $T_{m i n}$. Also, from the maximum distances we choose the minimum of the maximum distances to be $T_{\max }$. Finally, we divide this interval, i.e. $T=\left[T_{\min }, T_{\max }\right]$, into 30 partitions and these portions will from the thresholds. To construct SC, we will start from $T_{2}$ to be the first threshold as the first threshold is not providing sufficient information about the topology of the bins. Below, in figure (3), we are showing the building blocks of our proposed scheme.

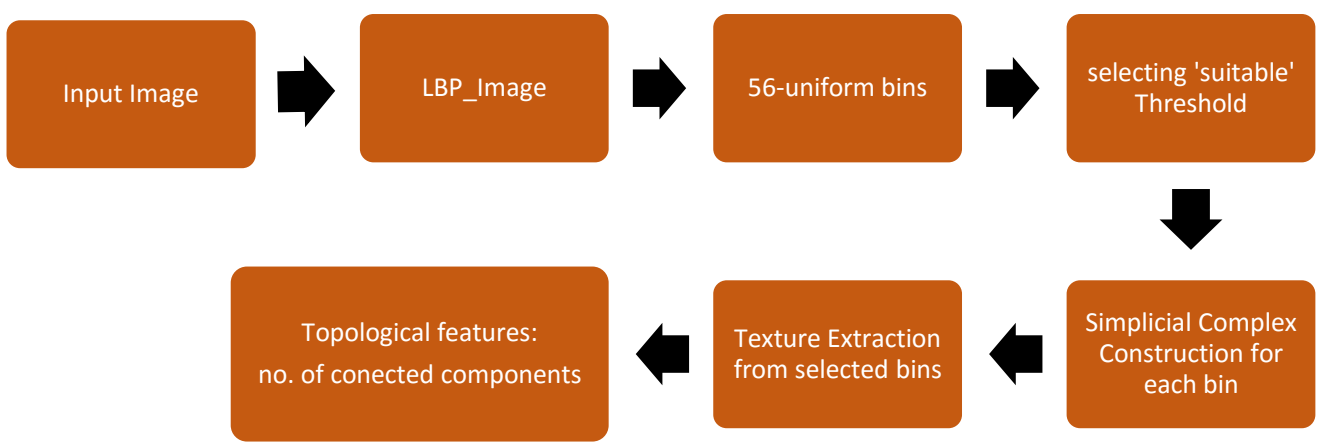

Figure (3): Simplicial complex construction for Image texture analysis from LBP.

\section{EXPERIMENTAL RESULTS}

To test our proposed scheme we used a well-known face database called Extended Yale B Database which contain 38 different persons, each with different lighting positions source. We selected 3 different set of all 38 persons, 3 different sets are clear normal faces, half-shadowed faces and full shadowed faces. Figure (4), shows an example of the face images which have been used for the experiments purpose. After building the simplicial complexes from the bins, we analyses carefully obtained patterns by looking at the local binary pattern codes, as a result we conclude that the cases where we have only 2 or 7 ones in the LBP-code patterns, distinguishing normal clear faces from 'shadowed' faces (half and full shadowed). As discussed in the previous section, we start from the second threshold and we compute the number of 
connected components of the Normal faces compared with half-shadow face images in the case of 2 and 7 ones in the LBP code patterns, which have been illustrated in figure (4).
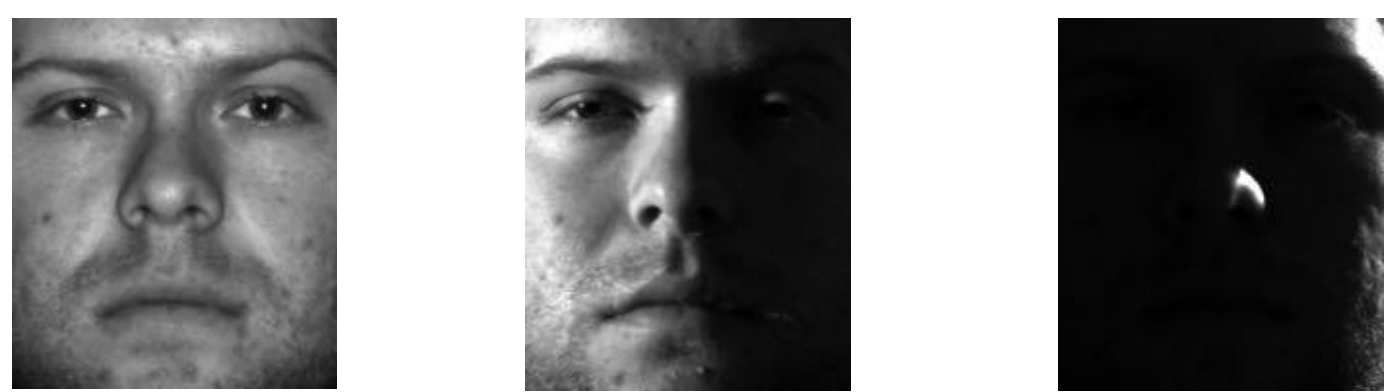

Figure (4): example of (Right) normal (Middle) half-shadow and (Left) full-shadow face images from Extended Yale B Database
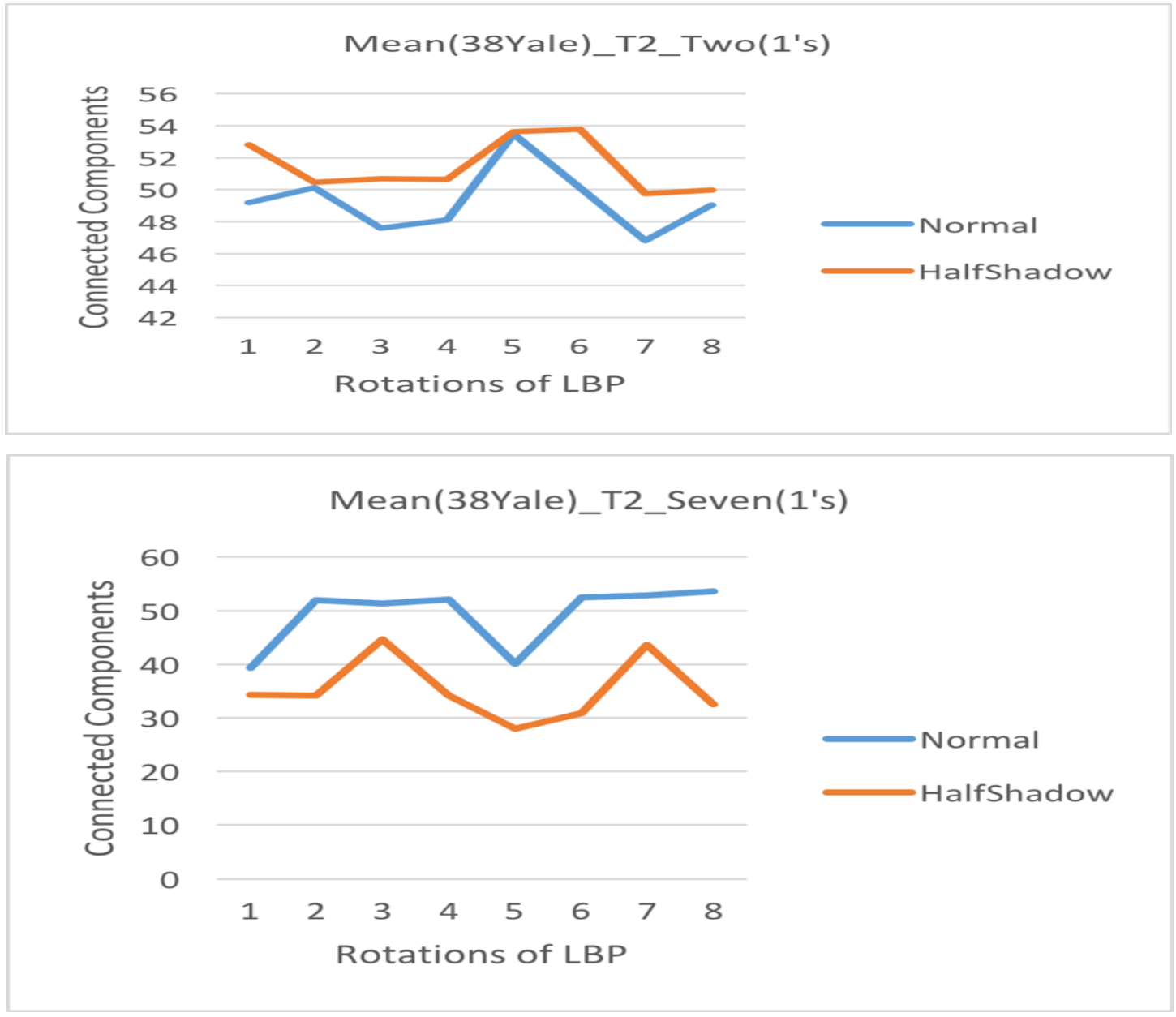

Figure (5): Connected Component results of Normal and half-shadow face images at $\mathrm{T}_{2}$. 
In figure (5), it is clear that the number of connected components, which have been calculated from SC of the bins where we have 2 and 7 ones in the LBP code patterns, of normal face images are different from half-shadowed face images. More precisely, in the case of having 7 ones in the LBP code pattern, the difference is significant and more distinguishable in comparison with the case where we have only 2 ones in the LBP code pattern.

Similar to half-shadow case, as it can be seen in figure (6), when we have 2 and 7 ones in LBP code patterns one can clearly distinguish between normal clear face images and full shadow faces. In addition, the cases where there is 3, 4 and 5 ones in the LBP code pattern, the normal face images are distinguishable from their full shadow counter parts. In the case of comparing normal faces with half shadow ones, the 3,4 and 5 ones in LBP codes are not as distinguishable as full shadow faces.
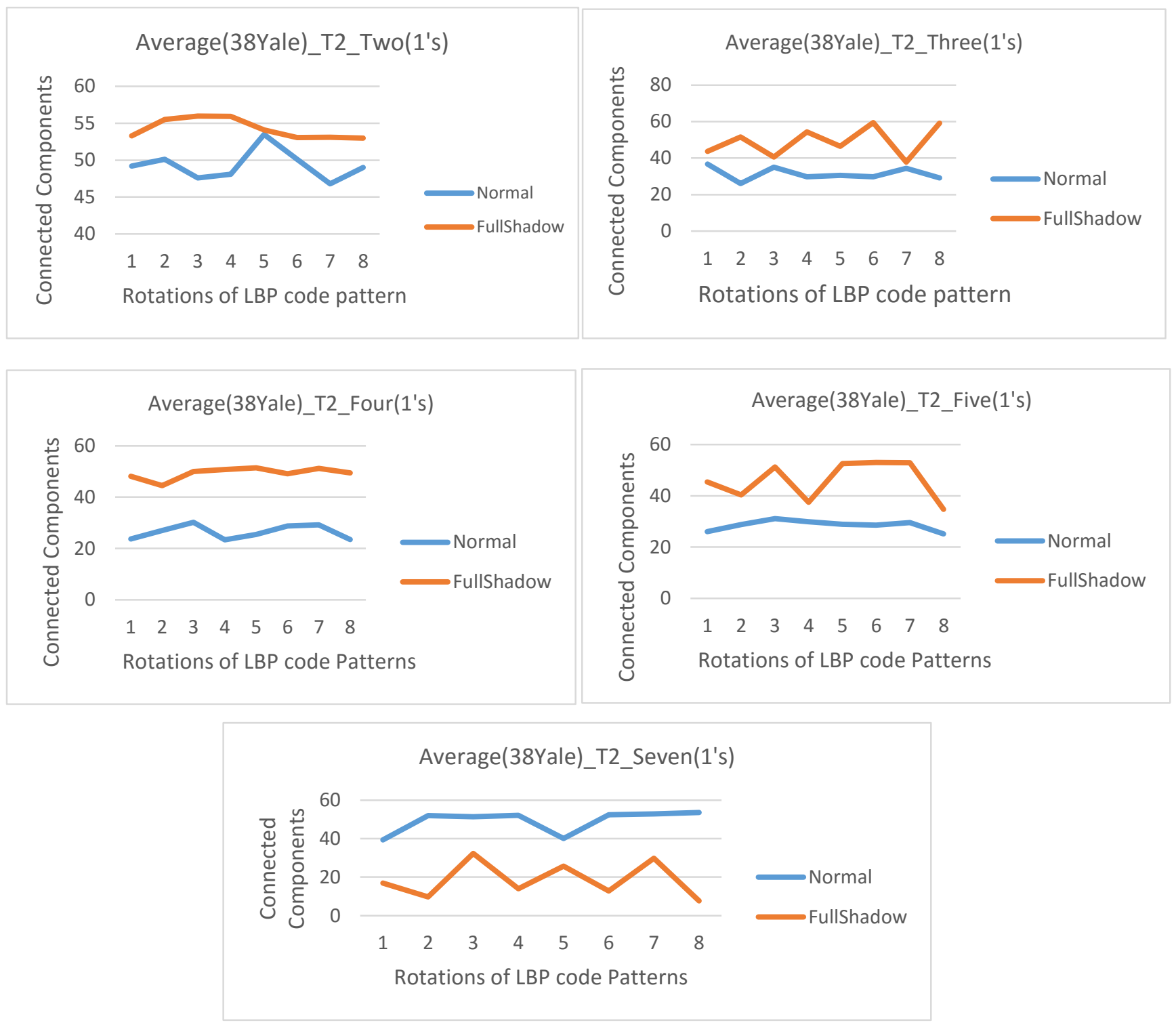

Figure (6): Connected Component results of Normal and full-shadow face images at $\mathrm{T}_{2}$. 
Increasing the threshold to $T_{3}$ will result in a consistent conclusion for half-shadow images apart from the case where there is seven ones in the LBP bin. On the other hand, for full-shadow images, increasing the threshold to $T_{3}$ will result in obtaining a consistent conclusion when there is two-ones and seven-ones in LBP bins.

Therefore, the number of connected components of simplicial complexes show a good indication for detecting the presence of shadow in face images especially in some of the uniform bins. Thus, we can conclude that not all of LBP-uniform bins are useful for detecting shadow. The rest of the five groups of LBP code patterns were also affected by adding shadow but they are not as distinguishable as the two groups that have been mentioned earlier.

This approach not only distinguishes different quality images, but it can also be used to detect the regions effected by shadow presence in the face images. As it can be seen in figure (7), constructed simplicial complexes can convey useful information (features) about normal, half-shadow and full-shadow .

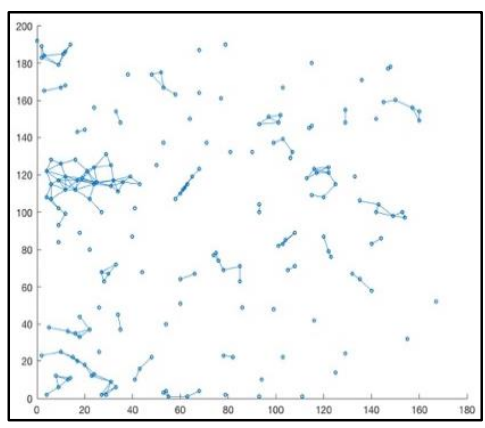

(a): Normal face image

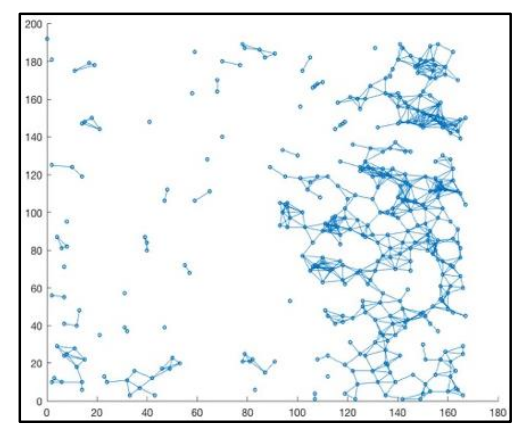

(b)half-shadow and

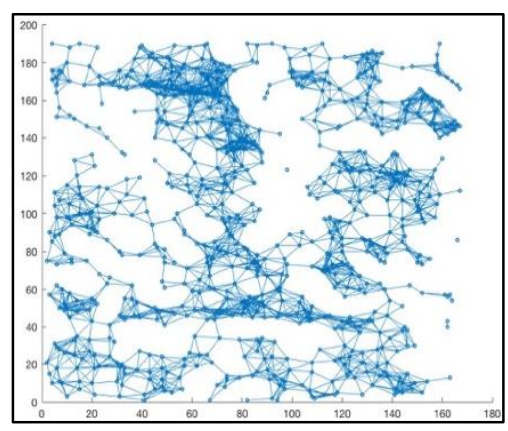

(c) full-shadow face images.

Figure (7): Simplicial Complex for LBP-Uniform bin 1011111.

\section{CONCLUSIONS}

Simplicial complex has been introduced as a fundamental tool to understand the shape or characteristics of complex data sets. Local binary pattern uniform bins have been used as a texture descriptor base to build the simplicial complexes for LBP bins which convey useful information about face images generally. Persistent homology invariants (e,g. number of connected components) was used to assess the degradation level and consequently analyzing the quality of image textures. Experimental results showed that persistent homology invariants, such as connected components, can detect the presence of shadow as well as detecting the infected regions by degradation. In future, we will extend our investigation to other types of degradation and consequently analysis the quality of images under these effects. 


\section{REFERENCES}

[1] P. Mohammadi, A. Ebrahimi-Moghadam and S. Shirani, "Subjective and Objective Quality Assessment of Image: A Survey," Majlesi Journal of Electrical Engineering, vol. 9, no. 1, pp. 5583, 2015.

[2] D. M. Chandler, "Seven Challenges in Image Quality Assessment: Past, Present, and Future Research," International Scholarly Research Notices, vol. 2013, 2013.

[3] Timo Ojala; Matti Pietikainen;David Harwood, "A comparative study of texture measures with classification based on featured distributions," Pattern Recognition, vol. 29, no. 1, pp. 51-59, 1996.

[4] R. D. Rashid, "Robust Steganographic Techniques for Secure Biometric-based Remote Authentication," Dphil in Computing, The University of Buckingham, Buckingham/United Kingdom, 2016.

[5] T. Ahonen; A. Hadid; M. Peitikainen, "Face description with local binary patterns: Application to face recognition," IEEE Transactions on Pattern Analysis and Machine Intellegence, vol. 28, no. 12, pp. 2037-2041, 2006.

[6] A. T. , H. A. and P. M., "Face recognition with Local Binary Patterns," 2004.

[7] R. D. Rashid, S. A. Jassim and H. Sellahewa, "LBP based on multi wavelet sub-bands feature extraction used for face recognition," South Hampton / United Kingdom, 2013.

[8] T. F. Majeed, "Segmentation, Super-resolution and Fusion for Digital Mammogram Classification," DPhil in Computing / The University of Buckingham, Buckingham / United Kingdom, 2016.

[9] P. Y. Lum, G. Singh, . A. Lehman, T. Ishkanov, M. Vejdemo-Johansson, . M. Alagappan, . J. Carlsson and G. Carlsson, "Extracting insights from the shape of complex data using topology," Nature, 2013.

[10] J. Lamar-Leon, E. B. Garcia-Reyes and R. Gonzal, "Human Gait Identification Using Persistent Homology," Progress in Pattern Recognition, Image Analysis, Computer Vision, and Applications, vol. 7441, pp. 244-251, 2012.

[11] J. Lamar Leon, A. Cerri and E. G. Reyes, "Gait-Based Gender Classification Using Persistent Homology," Progress in Pattern Recognition, Image Analysis, Computer Vision, and Applications, vol. 8259, no. 2, pp. 366-373, 2013.

[12] J. Lamar Leon, R. Alonso, E. Garcia Reyes and R. Gonzalez Diaz, "Topological Features for Monitoring Human Activities at Distance," Activity Monitoring by Multiple Distributed Sensing, vol. 8703, pp. 40-51, 2014.

[13] P. Bendich, J. S. Marron, E. Miller, A. Pieloch and S. Skwerer, "Persistent homology analysis of brain artery trees," The Annals of Applied Statistics, vol. 10, no. 1, pp. 198-218, 2016.

[14] B. Sreeparna, "Size functions in galaxy morphology classification," International Jornal of computer applications, vol. 100, no. 3, pp. 1-4, 2014.

[15] B. Rieck and H. Leitte, "Persistent Homology for the Evaluation of Dimensionality Reduction Schemes," 2015. 
[16] A. A. D. Rubin and G. Carlsson, "Classification of hepatic lesions using the matching metric," Computer vision and image understanding, vol. 121, pp. 36-42, 2014.

[17] I. Janusch and W. G. Kropatsch, "Shape classification using LBP and persistent of Critical Points," Lecture Notes in Computer Science, vol. 9647, 2016.

[18] C. Gunnar, "Topology and data," Bulletin of the American Mathematical Society, vol. 46, no. 2, pp. 255-308, 2009.

[19] H. Edelsbrunner and D. Morozov, "Persistent homology: Theory and practice," in In: European Congress of Mathematics Kraków, 2-7 July 2012. 